\title{
Geographische Umweltmessnetzanalyse und -planung
}

\section{Winfried Schröder, Roland Pesch, Vechta}

\section{Hintergrund und Ziele}

Die Umweltbeobachtung von Bund und Ländern wird in Deutschland seit $2003 \mathrm{im} \S 12$ des Bundesnaturschutzgesetzes geregelt. Sie soll methodisch harmonisierte, vergleichbare Daten liefern, die sich ohne thematische Lücken zielgerichtet und effizient zu einem Gesamtbild verknüpfen lassen. Obwohl die meisten Umweltdaten mit terrestrischen Verfahren als räumliche Stichproben und nicht flächendeckend mittels Fernerkundung erhoben werden, sollen sie ohne geographische Fehlstellen und möglichst flächendeckend gültig sein.

$\mathrm{Ob}$ die Umweltbeobachtung diese Anforderungen erfüllt, bedarf der Überprüfung. Hierfür wurden ein elektronischer Fragebogen mit rund 800 Items und spezielle Auswertungswerkzeuge entwickelt (SCHRÖDER et al. 2001). Zur Überprüfung der geographischen Anforderungen dienen eine multivariat-statistische Raumgliederung sowie geostatistische Verfahren, die wie die Metadatenbank mit einem Geographischen Informationssystem (GIS) für integrative Analysen verknüpft sind. In dieser Arbeit werden die Raumgliederung und die Geostatistik dazu benutzt, die räumlichen Strukturen der in Moosen gemessenen Metallakkumulation im Zeitverlauf flächenbezogen zu analysieren. Hierzu wird das genannte Instrumentarium mit der Berechnung einer Maßzahl der Metallakkumulation verknüpft. Dieser Index soll zur Kennzeichnung regionaler Akkumulationstypen sowie für Zeitreihenanalysen geeignet sein. Eine Übersicht der in diesem Artikel erarbeiteten Ergebnisse sowie der damit verbundenen methodischen Verfahrensschritte gibt die Abbildung 1.

\section{Methoden}

\subsection{Raumgliederung und Stoffhaushaltsmessungen unter ökotoxikologischem Aspekt}

Zur räumlichen Differenzierung der ökosystemaren Stoff- und Energieflüsse dienen meist zwei komplementäre Verfahrensansätze: die landschaftsökologische Kartierung (MARKS et al. 1989) und die seit den 1970er Jahren zunehmend mögliche geochemische Quantifizierung des Stoffhaushalts (FRÄNZLE 1998). Ziel der Messungen sind die Bestimmung des Stabilitätsfeldes ökologischer Systeme sowie die darin eingebettete Abschätzung der Umweltkonzentration von Stoffen (predicted environmental concentration, PEC), deren Abgleich mit ökotoxikologischen Wirk- schwellen (predicted no effect concentrations, PNEC) eine Einstufung stofflicher Umweltrisiken ermöglicht (FräNZLE et al. 1995). Die Kombination der landschaftsökologischen Raumgliederung mit der Bestimmung der Umweltkonzentration von Stoffen ist im Zusammenhang mit den in Kapitel 1 genannten Anforderungen an die Umweltbeobachtung nach $\$ 12$ Bundesnaturschutzgesetz praktisch und wissenschaftlich bedeutsam.

\subsection{Landschaftsökologische Raumgliederung}

Umweltbeobachtung ohne "geographische Fehlstellen» meint, dass die Umweltbeobachtungsmessnetze die räumliche Struktur von wichtigen, zu Landschaftseinheiten aggregierten physisch-geographischen Merkmalen terrestrischer Ökosysteme flächenrepräsentativ abdecken. Dies ist sinnvoll, weil jede Strukturbildung das Ergebnis der mit Energieflüssen verknüpften räumlichen Verlagerung bzw. Organisation von Materie ist (White et al. 1984: 29-34, 313). Während in geschlossenen Systemen die Entropie maximiert und die arbeitsfähige Energie (Exergie) minimiert wird, erreichen offene Systeme wie Ökosysteme oder Ökosystemkomplexe (Landschaften) dieses thermodynamische Gleichgewicht maximaler Entropie nicht. Vielmehr ist der wahrscheinlichste Zustand offener Systeme derjenige, in dem die Entropieproduktion pro Energietransfer-Einheit minimiert wird. Dies wird durch Strukturierung (Negentropie-Bildung, Organisation) im System und komplementären EntropieExport erreicht. Dieser Zustand wird als stationäres Fließgleichgewicht bezeichnet und kann nur bei permanentem Energie- und Stofffluss aufrechterhalten werden. Die horizontale und vertikale Differenzierung von Vegetationskomplexen ist ein Beispiel dieser Negentropie-Bildung offener Systeme und Ausdruck der damit optimierten Nutzung von Energie-, Wasserund Nährstoffressourcen. Das für die Wechselbeziehung zwischen Phytozönosen und Biotopen beispielhaft Dargelegte gilt auf allen räumlichen Skalen bis hin zum Landschaftsmaßstab. Insofern sind Landschaftsstrukturen Komplexindikatoren für die stofflichen und energetischen Randbedingungen, unter denen Ökosysteme funktionieren. Deshalb sollte die räumliche Struktur von Umweltmessnetzen an den Landschaftsstrukturen orientiert, die Daten der Umweltbeobachtung mit den landschaftsökologischen Basisdaten verknüpft werden. Für diese beiden Aspekte wird eine aus flächendeckenden Daten zu räumlichen Strukturen landschaftsökologischer Merkmale statistisch abgeleitete Raumgliederung benötigt, die in einem Geographischen Informationssystem (GIS) mit den Messdaten und den dazu gehörenden 


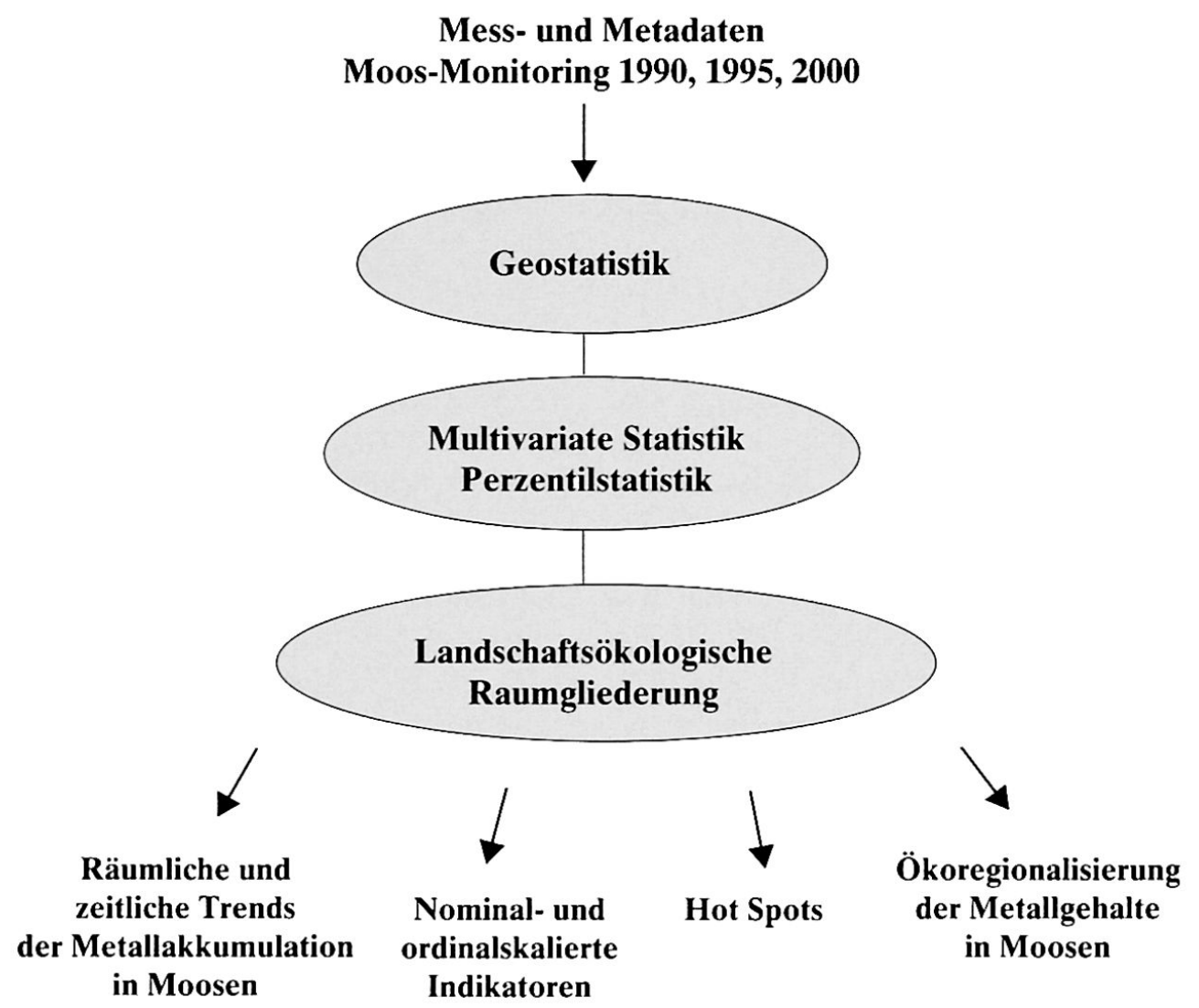

Abb. 1: Verfahrensgang der geographischen Messnetzanalyse der Moos-Monitoring-Kampagnen 1990, 1995 und 2000

Procedure applied in the geographical monitoring net analysis of the moss monitoring surveys of 1990,1995 and 2000 Processus de l'analyse géographique de réseau de mesure des campagnes de "monitoring" de mousse (1990, 1995 et 2000)

Metadaten verknüpft werden kann. Die von MEYNEN et al. (1953,1962) und RENNERS (1991) vorgelegten Raumgliederungen erfüllen diese Anforderungen historisch bedingt nicht. Weitere Raumgliederungsansätze diskutieren u.a. Bailey (1996), KliJN (1997), Müller (1981), Schröder (1994), Schulz (2000) sowie SteINHARDT und VOLK (1999). Die Datengrundlagen und die Verfahrensstruktur der in der vorliegenden Arbeit vorgestellten multivariat-statistischen Raumgliederung sind in Abbildung 2 (aufgeschaltet unter http:// people.freenet.de/wschroeder/) dargestellt.

Die potenziell natürliche Vegetation ( $\mathrm{pnV}$ ) kennzeichnet das ökologische Potenzial eines Raumes. Jedoch bedarf die mit der pnV-Karte Europas (BoHN et al. 2003) vorliegende ökologische Raumgliederung einer Ergänzung durch die Verknüpfung der pnVLegendeneinheiten mit ihren physisch-geographischen Bedingungsfaktoren (Globalstrahlung, Niederschlag, Luftemperatur, Verdunstung, Bodenarten und orographische Höhe). Hierzu wurde die Karte der pnV Deutschlands mit den 47 Karten über die phy- sisch-geographischen Elementarfaktoren in einem GIS verknüpft und die Zusammenhänge mit Classification and Regression Trees (CART; BREIMANN et al. 1984) analysiert. Hierzu wird jede der 48 Karten in 88.400 Zellen mit $2 \mathrm{~km} \times 2 \mathrm{~km}$ Kantenlänge zerlegt. Die Zellen werden mit CART nach der Ähnlichkeit der Ausprägung der pnV und ihrer physisch-geographischen Faktoren räumlich klassifiziert. Hierfür müssen alle Daten in einer Tabelle zusammengeführt werden. Diese umfasst 88.400 Zeilen und neben den geographischen Koordinaten entsprechend der Merkmalsanzahl 48 Spalten. CART zerlegt die Ausgangsmenge der Rasterzellen in möglichst wenigen binären Schritten in Untergruppen, die hinsichtlich der pnV und ihrer Bedingungsfaktoren möglichst homogen sind. Hierzu prüft CART: 1. ob die Rasterzellen mit derselben Ausprägung der Zielvariablen ( $\mathrm{pnV}$ ) durch identische Ausprägungen der Begleitmerkmale beschrieben sind, 2. ob die Objekte mit unterschiedlichen Ausprägungen der Zielvariablen verschiedene Ausprägungen der Begleitmerkmale aufweisen. Wenn Randbedingung 1 zutrifft, dann werden die Zellen einer Gruppe zuge- 
ordnet. Trifft sie nicht zu, werden die Zellen in verschiedene Klassen aufgeteilt. Ist Randbedingung 2 erfüllt, werden die Zellen verschiedenen Klassen zugewiesen. Ist Randbedingung 2 nicht gegeben, gelangen die Zellen in eine Gruppe. Insgesamt steigt so der Anteil der Zellen mit der in der jeweiligen Ausgangsmenge häufigsten Ausprägung der Zielvariablen schrittweise. CART vergleicht alle Möglichkeiten einer Gliederung miteinander und schlägt die statistisch optimale vor. Diese umfasst im vorliegenden Falle 73 Klassen. Zum Vergleich sei erwähnt, dass die Raumgliederung anhand der pnV 67 Klassen (BoHN et al. 2003), die Naturraumgliederung nach MEYNEN et al. $(1953,1962) 89$ Gruppen naturräumlicher Haupteinheiten und die geoökologische Gliederung von ReNNERS (1991) 84 Raumeinheiten unterscheiden.

Von den 73 landschaftsökologischen Klassen gelangen 21 in Abbildung 2 (aufgeschaltet unter http:// people.freenet.de/wschroeder/) zur Darstellung. Für jede der Raumklassen lässt sich die statistische Verteilung der zur Gruppierung verwendeten Merkmale grafisch darstellen und statistisch beschreiben (HoRNSMANN 2003; SCHMIDT 2002).

Die statistische Beschreibung der ökologischen Eigenschaften der Raumklassen unterstützt die Interpretation von Daten über die Umweltkonzentration (PECDaten) von Stoffen. Die mit der nassen und trockenen Deposition eingetragenen Metalle werden in den Moosen wie in anderen Pflanzen zwischengespeichert, bevor sie durch Auswaschung oder Remineralisierung der Biomasse in den Boden gelangen. Die Messdaten des Moos-Monitorings und die daraus geostatistisch geschätzten Flächendaten lassen sich im GIS mit denjenigen Gebieten der landschaftsökologischen Raumklassen in Anlehnung an HENNINGS (2000) verknüpfen, in denen die Schwermetallbindung in den Böden gering und die Wahrscheinlichkeit ihres Übertritts in das Grundwasser hoch ist. Diese Risikolokalisierung lässt sich anhand der Messungen einschlägiger Umweltbeobachtungsnetze empirisch validieren, deren Identifizierung und Lokalisierung mit einer an das GIS gekoppelten Metadatenbank erfolgt (Weustermann 2003).

\subsection{Erfassung der Metallakkumulation in Moosen}

Die Umweltkonzentration (PEC) von Metallen in den landschaftsökologisch definierten Teilräumen Deutschlands lässt sich durch die geochemische Bestimmung der Metallgehalte in Moosen einer räumlichen Stichprobe quantifizieren. Moose haben eine große spezifische Oberfläche, welche die trocken oder nass deponierten Metalle über mehrere Jahre ohne physiologische und strukturelle Veränderungen akkumulieren. Die Anreicherung der Metalle in den Moosen erhöht die Präzision der geochemischen Elementbestimmung. Hinzu kommen enorme finanzielle Vorteile bioindikato- rischer Methoden gegenüber technischen Verfahren zur Quantifizierung von Metalleinträgen. Deshalb erfolgt das Moos-Monitoring nach erfolgreichen Pilotanwendungen in Skandinavien zwischen 1970 und 1985 seit 1990 alle fünf Jahre zeitgleich in 21 bis 28 europäischen Staaten (BUSE et al. 2003). Die Moosproben wurden in Deutschland 1990 an 592, 1995 an 1026 und im Jahr 2000 an 1028 Standorten nach UNECE (2001) entnommen (Herpin et al. 1995; Siewers \& HerPin 1998; SIEWERS et al.2000; SchröDER et al.2002). Eine Übersicht der räumlichen Verteilung der Messstandorte inkl. der dort gemessenen $\mathrm{Zn}$-Akkumulationen gibt die Abbildung 3 (aufgeschaltet unter http://people.freenet.de/ wschroeder/). Dabei hat die Beprobung von Pleurozium schreberi (P.s.) höchste Priorität, es folgen abgestuft Scleropodium purum (S.p.) und Hypnum cupressiforme (H.c.). Werden diese prioritären Moose nicht gefunden, sind Ersatzarten zu entnehmen. Ökologisch wesentliche Merkmale der Probenentnahmeorte sind in einem Access-Datenbankformular festzuhalten.

Die Bestimmung der Akkumulation von mindestens 12 Metallen (As, Cd, Cr, Cu, Fe, Hg, Ni, Pb, Sb, V, Ti, $\mathrm{Zn}$ ) erfolgt qualitätskontrolliert mit ICP-MS (inductively coupled plasma - mass spectrometer), ICP-OES (inductively coupled plasma - optical emission spectrometer) sowie AAS (Atomabsorptionsspektrometer)Kaltdampf-Technik. Die Vergleichbarkeit der Analysendaten der Moos-Monitoring-Kampagnen 1990, 1995 und 2000 wurde anhand von Rückstellproben mit gutem Ergebnis festgestellt.

\subsection{Räumliche Verallgemeinerung der Stichproben- daten}

Aufgrund der schiefen Messwertverteilungen wurden neben den Originaldaten auch die lognormal-transformierten Messdaten geostatistisch ausgewertet (SCHRÖDER et al. 2002). Alle berechneten Variogramme zeigen z.T. deutliche räumliche Autokorrelationen in den ersten Distanzklassen, doch sind bei fast jedem Element hohe Nugget-Effekte festzustellen. Letztere sind auf große Messwertunterschiede in den ersten Distanzklassen zurückzuführen. Dies untermauert die von SiEwers et al. (2000) nachgewiesene Variabilität der Metallgehalte in den Moosproben an jeweils einem Probenentnahmestandort. Die Autokorrelationsreichweiten betragen bei den Originaldaten von $33 \mathrm{~km}(\mathrm{Fe})$ bis zu $169 \mathrm{~km}(\mathrm{Zn})$ und bei den lognormal-transformierten Daten von $89 \mathrm{~km}(\mathrm{~V})$ bis zu $248 \mathrm{~km}(\mathrm{Hg})$.

GIS-Technik und Geostatistik ermöglichen die Berechnung verschiedener Schätzgütekriterien. Die Verfahren hierfür sind die Kreuzvalidierung und der MesswertRasterwertvergleich (SCHRÖDER et al. 2002).

- Bei der Kreuzvalidierung wird aus dem Gesamtdatensatz nacheinander je ein Messwert entnommen und durch Kriging anhand eines Modell- 


\begin{tabular}{|l|l|}
\hline $\begin{array}{l}\text { Mittlerer absoluter } \\
\text { Fehler (MAE) }\end{array}$ & $\begin{array}{l}\text { Zur Berechnung des mittleren absoluten Fehlers werden die Beträge der Abweichungen } \\
\text { zwischen Messwert und Schätzwert gemittelt. Dadurch wird das Egalisieren von } \\
\text { negativen und positiven Werten verhindert, und man erhält ein Maß dafür, wie stark die } \\
\text { geschätzten von den gemessenen Werten abweichen. }\end{array}$ \\
\hline $\begin{array}{l}\text { Mittlerer prozentualer } \\
\text { Fehler (MPE) }\end{array}$ & $\begin{array}{l}\text { Der mittlere absolute Fehler sagt direkt nichts darüber aus, wie hoch die relativen } \\
\text { Abweichungen zwischen Mess- und Schätzwerten sind. Setzt man den jeweiligen } \\
\text { Messwert gleich 100\%, so kann die Differenz zwischen Mess- und Schätzwert in Prozent } \\
\text { angegeben werden. Der mittlere prozentuale Fehler ist der Mittelwert der so } \\
\text { berechneten prozentualen Abweichungen. Schätzungen unterschiedlicher Messgrößen } \\
\text { können so vergleichbar gemacht werden. }\end{array}$ \\
\hline $\begin{array}{l}\text { Korrelationskoeffizient } \\
\text { nach Pearson (K) }\end{array}$ & $\begin{array}{l}\text { OLEA (1999) schlägt den Korrelationskoeffizienten nach PEARSON zwischen } \\
\text { Mess- und Schätzergebnissen als Gütekennzahl vor. } \\
\text { Dieser ist im Idealfall 1. }\end{array}$ \\
\hline
\end{tabular}

Tab. 1: Definitionen der Qualitätsmasse für Kriging-Flächenschätzungen

Definition of qualitative variables used in Kriging estimates of surface area

Définitions des variables qualitatives utilisées pour les évaluations Kriging de surfaces

Variogramms neu geschätzt. Die Abweichung des Schätzwertes vom Messwert ist ein $\mathrm{Maß}$ für die Schätzqualität. Die Differenzen zwischen Mess- und Schätzwerten können anschließend durch statistische Kennzahlen beschrieben werden (Tabelle 1).

- Beim Messwert-Rasterwert-Vergleich werden die Messpunkte mit den jeweiligen Flächenkarten im GIS verschnitten. Jeder Messpunkt wird dabei mit einem Rasterzellenwert verglichen. Die Differenzen zwischen Mess- und Rasterwerten werden anschließend wie bei der Kreuzvalidierung statistisch beschrieben. Durch die Verschneidung von Punktund Rasterdaten im GIS können die Messwerte den Raster- bzw. Schätzwerten gegenübergestellt werden. Hier werden alle Messwerte mit allen Schätzwerten «simultan» verglichen. Dabei muss berücksichtigt werden, dass ein Rasterwert eine räumliche Verallgemeinerung des Mittelpunkts einer Rasterzelle darstellt und insofern mit geometrischen Ungenauigkeiten behaftet ist: Bei dem hier verwendeten Raster von $3 \times 3 \mathrm{~km}^{2}$ kann der Messpunkt maximal $2.1 \mathrm{~km}$ von dem geschätzten Punkt entfernt liegen. Die so ermittelten Messwert-Rasterwert-Differenzen können wie bei der Kreuzvalidierung mit Hilfe von Schätzgütekennzahlen zusammengefasst werden (Tabelle 1).

Die Ergebnisse der Kreuzvalidierung sowie des Messwert-Rasterwertabgleichs aus dem Moos-Monitoring 2000 sind den Tabellen 2 und 3 zu entnehmen. Gemessen an den mittleren prozentualen Fehlern der Kreuzvalidierung weisen die Kriging-Ergebnisse der Originaldaten eine unbefriedigende Schätzqualität auf. Für $\mathrm{Al}, \mathrm{As}, \mathrm{Fe}, \mathrm{Ti}$ und $\mathrm{Sb}$ liegen die Unsicherheiten über $40 \%$. Die Korrelationskoeffizienten liegen alle unter
0.6, bei Fe,Ti und V unter 0.5. Die Schätzgüteparameter der Messwert-Rasterwertvergleiche weisen bessere Werte auf. So liegen die mittleren prozentualen Fehler mit Ausnahme von As unter 30\%. Die Korrelationen zwischen Mess- und Rasterwerten rangieren mit Ausnahme von $\mathrm{Cd}(\mathrm{K}=0.68)$ über 0.8 .

Im Vergleich der Kreuzvalidierungsergebnisse weisen die Originaldaten zum größten Teil bessere Werte auf als die lognormal-transformierten Daten. Dies äußert sich vor allem bei den mittleren absoluten und prozentualen Schätzwerten. Für As und Ti können Unterschiede von über $10 \%$, bei $\mathrm{Sb}$ von knapp $20 \%$ nachgewiesen werden, bei $\mathrm{Cd}, \mathrm{Cr}, \mathrm{Cu}, \mathrm{Hg}, \mathrm{Ni}$ und $\mathrm{Zn}$ betragen sie unter 3\%. Die Korrelationen sind gegenüber den Originaldaten zum Teil stärker ausgeprägt, doch liegen diese bis auf $\mathrm{Ni}(=0.6)$ unter 0.6. Die Schätzgüteparameter des MesswertRasterwertvergleichs weisen keine eindeutigen Tendenzen zugunsten der lognormal-transformierten Kriging-Ergebnisse auf. $\mathrm{Bei} \mathrm{Al}, \mathrm{As}, \mathrm{Cd}$ und $\mathrm{Zn}$ sind niedrigere mittlere prozentuale Fehler festzustellen. Bis auf $\mathrm{Cd}$ zeigen sich schwächere Korrelationen als bei den Originaldaten, doch liegen diese in allen Fällen über 0.75 .

Alle für 1990, 1995 und 2000 mit Ordinary Kriging berechneten Flächenschätzungen der Metall-Akkumulation wurden mit Hilfe von ArcView GIS kartografisch aufgearbeitet (PESCH 2003). Die Abbildung 4 (aufgeschaltet unter http://people.freenet.de/ wschroeder/) zeigt das Beispiel Zn, dessen Hauptemissionsquellen auf die Produktion und Veredelung von Zink, andere Buntmetallindustrien sowie auf Kohlekraftwerke zurückzuführen sind (SIEwERS \& HerpiN 


\begin{tabular}{|l|c|c|c|c|c|c|c|}
\hline \multirow{2}{*}{} & \multirow{2}{*}{$\mathbf{n}$} & \multicolumn{3}{|c|}{ Originaldaten } & \multicolumn{3}{c|}{ Ln-transformiert } \\
\cline { 3 - 8 } & $\begin{array}{c}\text { MAE } \\
{[\boldsymbol{\mu g} / \mathbf{g}]}\end{array}$ & $\begin{array}{c}\text { MPE } \\
{[\%]}\end{array}$ & $\mathbf{K}^{*}$ & $\begin{array}{c}\text { MAE } \\
{[\boldsymbol{\mu g} / \mathbf{g}]}\end{array}$ & $\begin{array}{c}\text { MPE } \\
{[\%]}\end{array}$ & $\mathbf{K}^{*}$ \\
\hline $\mathbf{A l}$ & 1028 & 151,1 & 41,5 & 0,55 & 134 & 33,3 & 0,58 \\
\hline $\mathbf{A s}$ & 1026 & 0,084 & 47,0 & 0,51 & 0,08 & 35,1 & 0,56 \\
\hline $\mathbf{C d}$ & 1027 & 0,064 & 27,4 & 0,5 & 0,06 & 24,5 & 0,54 \\
\hline $\mathbf{C r}$ & 1025 & 0,264 & 25,9 & 0,56 & 0,25 & 23,3 & 0,58 \\
\hline $\mathbf{F e}$ & 1026 & 154,2 & 40,8 & 0,44 & 133 & 31,4 & 0,52 \\
\hline $\mathbf{C u}$ & 1027 & 1,54 & 21,6 & 0,55 & 1,52 & 20,6 & 0,54 \\
\hline $\mathbf{H g}$ & 1028 & 0,012 & 26,9 & 0,55 & 0,01 & 24,8 & 0,55 \\
\hline $\mathbf{N i}$ & 1028 & 0,382 & 31,2 & 0,59 & 0,37 & 27,4 & 0,6 \\
\hline $\mathbf{P b}$ & 1026 & 1,882 & 37,8 & 0,53 & 1,77 & 31,2 & 0,54 \\
\hline Ti & 1023 & 4,13 & 47,3 & 0,49 & 3,73 & 36,5 & 0,54 \\
\hline Sb & 1028 & 0,085 & 47,0 & 0,51 & 0,04 & 28,4 & 0,55 \\
\hline $\mathbf{V}$ & 1027 & 0,409 & 37,7 & 0,48 & 0,39 & 32,5 & 0,45 \\
\hline Zn & 1026 & 10,32 & 23,9 & 0,53 & 10,1 & 22,3 & 0,53 \\
\hline
\end{tabular}

*Alle Korrelationskoeffizienten sind auf dem 1\%-Niveau signifikant.

Tab. 2: Ausgewählte Kennziffern der Kreuzvalidierung des Moos-Monitorings 2000

Selected criteria used in the cross-validation of moss monitoring in 2000

Choix d'indices de validation contradictoire du «monitoring» de mousse 2000

1998). Es wird weiterhin vermutet, dass über den Reifenabrieb und die Kraftstoffverbrennung erhebliche Mengen an Zink in die Atmosphäre gelangen (NriagU \& PaCyna 1988; Ohnsorge \& Wilhelm 1991). Abbildung 4 zeigt die Entwicklung der $\mathrm{Zn}$-Akkumulation in den Moosen sehr viel deutlicher als die Punktkarten der Abbildung 3: Von 1990 bis 1995 stieg sie deutschlandweit an, während in der dritten Messkampagne (2000) die geringsten Konzentrationen festgestellt wurden. Mit den Flächenschätzungen können nun flächenhafte, mehrere Metalle integrierende Belastungsindikatoren berechnet werden (Kap. 3).

\section{Indizes der Metallakkumulation}

Für die Umwelt- und Nachhaltigkeitsberichterstattung sind umfangreiche Datenerhebungen weitgehend zusammenzufassen (PEICHL 2001). Hierzu werden die geostatistischen Flächenschätzungen der in den drei Monitoring-Kampagnen durchgängig auch in den neuen Bundesländern gemessenen acht Metalle $(\mathrm{Cr}$, $\mathrm{Cu}, \mathrm{Fe}, \mathrm{Ni}, \mathrm{Pb}, \mathrm{Ti}, \mathrm{V}, \mathrm{Zn}$ ) in zwei Indizes gebündelt (Рesch 2003). Zum einen werden die Probenentnahmestandorte nach z-Transformation entsprechend ihrer Ähnlichkeit in Bezug auf die Akkumulation der acht Metalle mit zwei clusteranalytischen Verfahren (Ward, kmeans; Mucha 1994) klassifiziert. Die Klassen bilden die Kategorien eines i.S.v. STEvens (1946) nominal skalierten Indexes, der regionale Akkumulationstypen abbildet (Kap. 3.2). Zum anderen liefert die Perzentil-Statistik der Schätzdaten einen ordinal skalierten Index, der besonders für die Abbildung der zeitlichen Trends der Metallakkumulation geeignet ist (Kap. 3.3). Er wird für die Identifizierung von Hot Spots der Metall-Akkumulation und zur naturräumlichen Differenzierung der Belastungsentwicklung verwendet.

\subsection{Regionale Akkumulationstypen}

Die Messstandorte der Erhebung 1990 und 1995 werden anhand der im Ward-Verfahren berechneten Fehlerquadratsumme optimal in fünf Klassen der Metallakkumulation gruppiert, im Jahr 2000 auf Grund geringerer räumlicher Varianz in vier Klassen. Abbildung 5 (aufgeschaltet unter http://people.freenet.de/ wschroeder/) zeigt das Ergebnis der clusteranalytischen Auswertung der Flächenschätzungen. In jeder Monitoring-Kampagne gibt es eine Klasse A mit Standorten deutschlandweit geringster Metallakkumulation. 


\begin{tabular}{|l|c|c|c|c|c|c|c|}
\hline \multirow{2}{*}{} & \multirow{2}{*}{} & \multirow{2}{*}{$\mathbf{n}$} & \multicolumn{3}{|c|}{ Originaldaten } & \multicolumn{3}{c|}{ Ln-transformiert } \\
\cline { 3 - 8 } & $\begin{array}{c}\text { MAE } \\
{[\boldsymbol{\mu g} / \mathbf{g}]}\end{array}$ & $\begin{array}{c}\text { MPE } \\
{[\%]}\end{array}$ & $\mathbf{K} *$ & $\begin{array}{c}\text { MAE } \\
{[\boldsymbol{\mu g} / \mathbf{g}]}\end{array}$ & $\begin{array}{c}\text { MPE } \\
{[\%]}\end{array}$ & $\mathbf{K} *$ \\
\hline $\mathbf{A l}$ & 1028 & 96,09 & 22,45 & 0,84 & 96,30 & 22,31 & 0,84 \\
\hline $\mathbf{A s}$ & 1026 & 0,058 & 31,99 & 0,85 & 0,058 & 25,56 & 0,77 \\
\hline $\mathbf{C d}$ & 1027 & 0,056 & 23,47 & 0,68 & 0,044 & 16,71 & 0,82 \\
\hline $\mathbf{C r}$ & 1025 & 0,10 & 10,14 & 0,96 & 0,14 & 12,30 & 0,92 \\
\hline $\mathbf{F e}$ & 1026 & 60,61 & 15,44 & 0,94 & 89,29 & 19,62 & 0,85 \\
\hline $\mathbf{C u}$ & 1027 & 1,15 & 15,99 & 0,82 & 1,37 & 18,24 & 0,66 \\
\hline $\mathbf{H g}$ & 1028 & 0,009 & 19,36 & 0,81 & 0,010 & 19,85 & 0,75 \\
\hline $\mathbf{N i}$ & 1028 & 0,24 & 19,64 & 0,90 & 0,26 & 19,05 & 0,84 \\
\hline $\mathbf{P b}$ & 1026 & 0,94 & 18,64 & 0,95 & 1,42 & 24,14 & 0,75 \\
\hline $\mathbf{T i}$ & 1023 & 1,60 & 17,42 & 0,95 & 2,75 & 24,78 & 0,81 \\
\hline $\mathbf{S b}$ & 1028 & 0,03 & 20,55 & 0,85 & 2,75 & 24,78 & 0,81 \\
\hline $\mathbf{V}$ & 1027 & 0,23 & 21,24 & 0,91 & 0,29 & 22,90 & 0,76 \\
\hline $\mathbf{Z n}$ & 1026 & 7,71 & 17,55 & 0,80 & 7,62 & 16,19 & 0,78 \\
\hline
\end{tabular}

* Alle Korrelationskoeffizienten sind auf dem 1\%-Niveau signifikant.

Tab. 3: Ausgewählte Qualitätskenngrössen des Messwert-Rasterwert-Abgleichs des Moos-Monitorings 2000 Selected qualitative factors applied in the raster value comparison of moss monitoring in 2000 Choix d'indices qualitatifs de grandeur, comparatifs de la valeur de trame, du «monitoring» de mousse 2000

Ferner wird das Ruhrgebiet jedes Mal durch vergleichsweise hohe Gehalte derjenigen Metalle gekennzeichnet, die vornehmlich dem Straßenverkehr zugeschrieben werden (1990 Cluster C, 1995 Cluster E, 2000 Cluster C). Schließlich kommen Standorte der Klasse D 1990 und 1995 überwiegend in SachsenAnhalt, Sachsen und Thüringen vor und weisen relativ hohe Gehalte an $\mathrm{Fe}, \mathrm{Ni}$, $\mathrm{Ti}$ und $\mathrm{V}$ aus Kohleund Braunkohlekraftwerken auf. Eine entsprechende Klasse wird in den Daten des Jahres 2000 nicht identifiziert, was durch die Reduktion der Emissionen erklärbar ist.

\subsection{Ordinaler Multi-Metall-Akkumulationsindex}

Kasten 1 enthält die Verfahrensschritte der Berechnung eines Indexes, der jede der $3 \times 3 \mathrm{~km}^{2} \mathrm{Ra}-$ sterflächen Deutschlands $(\mathrm{n}=40.963)$ Kampagnen und Metalle übergreifend hinsichtlich der Metallakkumulation kennzeichnet. Abbildung 6 (aufgeschaltet unter http://people.freenet.de/wschroeder/) zeigt eine deutschlandweite Abnahme der Metallakkumulation. Während $199042 \%$ der Fläche einen Akkumulationsindex $>8$ (rot) aufwies, waren es $199510 \%$. In 2000 waren derartige Flächen nicht nachweisbar.
Der Kampagnen und Metalle integrierende Akkumulationsindex kann für die Gesamtfläche Deutschlands und die landschaftsökologischen Raumklassen bestimmt werden. Wenn man die über alle drei Messkampagnen bundesweit gemittelte Metall-Akkumulation gleich $100 \%$ setzt und darauf die entsprechenden Indizes der ökologischen Raumklassen bezieht (Kasten 2), zeigt sich u.a. (Abbildung 7, aufgeschaltet unter http://people.freenet.de/wschroeder/): 1990 lag die Metallakkumulation in den Alpen zwischen -10 und $-20 \%$ unter dem Bundesdurchschnitt der drei Messkampagnen, das Alpenvorland wich hiervon um 0 bis $-10 \%$ ab. In Nordwestdeutschland differierte die Metallakkumulation um 0 bis $+10 \%$ vom Durchschnitt Deutschlands, während in allen anderen landschaftsökologisch definierten Teilräumen eine z.T. $+60 \%$ überschreitende Akkumulation von $\mathrm{Cr}, \mathrm{Cu}$, $\mathrm{Fe}, \mathrm{Ni}, \mathrm{Pb}, \mathrm{Ti}, \mathrm{V}$ und $\mathrm{Zn}$ auftrat. 1995 war die Metallakkumulation in den Alpen und im Alpenvorland um -20 bis $-60 \%$ geringer als im Bundesdurchschnitt der drei Kampagnen. Im Jahr 2000 betrug die Abweichung der Metallakkumulation in den Alpen im Vergleich zum Durchschnitt -70 bis $-80 \%$, in allen anderen Naturräumen mindestens $-20 \%$. 
1. Geostatistische Verallgemeinerung der Akkumulation jeweils von $\mathrm{Cr}, \mathrm{Cu}, \mathrm{Fe}, \mathrm{Ni}, \mathrm{Pb}, \mathrm{Ti}, \mathrm{V}$ und $\mathrm{Zn}$ für jede der drei Messkampagnen

\section{ZWISCHENERGEBNIS: 3 x 8 Flächenkarten à 40.963 Zellen (3 km x 3 km²)}

2. Export der kampagnenspezifischen Ergebnisse in je eine XYZ-Tabelle

3. Hinzufügen einer Spalte «Erhebungsjahr» und Füllung der Spalten mit dem Kampagnenjahr (1990 oder 1995 oder 2000)

4. Verknüpfung der drei Tabellen zu einer Tabelle

ZWISCHENERGEBNIS: 1 Tabelle à 11 Spalten (X-Koordinate; Y-Koordinate; $\mathrm{Cr}, \mathrm{Cu}, \mathrm{Fe}, \mathrm{Ni}, \mathrm{Pb}, \mathrm{Ti}, \mathrm{V}$ und $\mathrm{Zn}$-Schätzwerte; Jahr) und 122.889 Zeilen

5. Ordnung der Schätzwerte für $\mathrm{Cr}$ nach der Größe

6. Einteilung der Werte in 10 gleich große Gruppen (Perzentile)

7. Vergabe von Cr-Indizes (von 1 bis 10) für jede Zelle

8. Durchführung der Schritte 6 bis 8 für alle anderen Elemente

ZWISCHENERGEBNIS: 1 Tabelle mit 19 Spalten (X-Koordinate; Y-Koordinate; $\mathrm{Cr}, \mathrm{Cu}, \mathrm{Fe}, \mathrm{Ni}, \mathrm{Pb}, \mathrm{Ti}, \mathrm{V}$ und $\mathrm{Zn}$-Schätzwerte und elementspezifische Indizes; Erhebungsjahr) und 122.889 Zeilen

9. Bildung eines Mittelwertes über alle 8 elementspezifischen Indizes pro Zeile

ERGEBNIS: 1 Tabelle mit je 122.889 Zeilen, bestehend aus 19 Spalten inkl. einer Spalte mit Angaben zu den Messwerte und Kampagnen übergreifenden Metallindizes

10. Import der Tabelle ins GIS und kartographische Darstellung

Kasten 1: Kampagnen integrierender Multi-Metall-Akkumulationsindex

Survey on integrative multi-metal accumulation indices

Index intégrateur des campagnes d'accumulation multimétallique

Abbildung 7 zeigt die hoch verdichteten Informationen über die Akkumulation von Metallen. Die statistische Aggregation ermöglicht es, die Daten für eine umweltchemische und ökotoxikologische Interpretation aufzubereiten. Hierzu gehört auch die Lokalisierung von Hot Spots der gemessenen resp. (geostatistisch) geschätzten Umweltkonzentration (PEC) potenziell toxischer Stoffe. Diese PEC Hot Spots lassen sich dadurch lokalisieren, dass man den Metallakkumulationsindex für jede der Messkampagnen berechnet. Diejenigen Standorte, die in jeder der drei Messphasen einen Metallindex größer 6 haben, werden als PEC Hot Spots definiert und kartiert. Abbildung 8 (aufgeschaltet unter http://people.freenet.de/ wschroeder/) weist 73 Standorte bzw. $22 \%$ der bundesdeutschen Fläche als PEC Hot Spots mit einem Metallakkumulationsindex $>6$ und 12 Probenentnahmeorte bzw. $5 \%$ mit einem Index $>8$ aus. An diesen Schwerpunkten der Metallakkumulation sollten im Moos-Monitoring 2005 weitergehende Untersuchungen zum Transfer der Metalle aus den Moosen in die Böden und das Grundwasser durchgeführt werden. Hierbei ist darauf zu achten, ob die PEC-Werte die Wirkschwellen (PNEC) der jeweiligen Metalle überschreiten.

\section{Resümee und Ausblick}

In diesem Artikel wurde anhand der bundesweiten Moos-Monitoring-Kampagnen gezeigt, wie mit Hilfe von Geostatistik, GIS und multivariater Statistik punktuell aufgenommene Umweltdaten flächenhaft verallgemeinert und $\mathrm{zu}$ Umweltindikatoren verdichtet werden können. Die Fülle der Messdaten wurde stufenweise komprimiert, um die räumlichen und zeitlichen Entwicklungen der Metallakkumulation herauszupräparieren. Die stoffliche Differenzierung wurde durch einen nominal und einen ordinal skalierten Index reduziert. Die beiden Indizes integrieren die aus den Messdaten aller Metalle geostatistisch berechneten Flächeninformationen. Die räumlichen Trends wurden zu den 21 Klassen einer multivariat-statistisch berechneten landschaftsökologischen Raumgliederung flächenhaft verallgemeinert. Damit lässt sich der ordinale Akkumulationsindex landschaftlich differenziert zur durchschnittlichen Metallakkumulation des Jahrzehnts 1990 bis 2000 ins Verhältnis setzen. Somit ist die Möglichkeit gegeben, die zeitlichen Trends der Metallakkumulation naturräumlich 
1. Geostatistische Verallgemeinerung der Akkumulation jeweils von $\mathrm{Cr}, \mathrm{Cu}, \mathrm{Fe}, \mathrm{Ni}, \mathrm{Pb}, \mathrm{Ti}, \mathrm{V}$ und $\mathrm{Zn}$ für jede der drei Messkampagnen

\section{ZWISCHENERGEBNIS: 3 x 8 Flächenkarten à 40.963 Zellen ( 3 km x 3 km²)}

2. Verschneiden jeder 3 x 8 Flächenkarten mit der Raumgliederung (siehe Kapitel 2.2)

3. Export der kampagnenspezifischen Ergebnisse in je eine XYZ-Tabelle

4. Hinzufügen einer Spalte «Erhebungsjahr» und Füllung der Spalten mit dem Kampagnenjahr (1990 oder 1995 oder 2000)

5. Verknüpfung der drei Tabellen zu einer Tabelle

ZWISCHENERGEBNIS: 1 Tabelle mit 12 Spalten (X-Koordinate; Y-Koordinate; Cr, $\mathrm{Cu}, \mathrm{Fe}, \mathrm{Ni}, \mathrm{Pb}, \mathrm{Ti}, \mathrm{V}$ und Zn-Schätzwerte; Raumklasse; Jahr) und 122.889 Zeilen

6. Ordnung der Schätzwerte für $\mathrm{Cr}$ nach der Größe

7. Einteilung der Werte in 10 gleich große Gruppen (Perzentile)

8. Vergabe von $\mathrm{Cr}$-Indizes (von 1 bis 10) für jede Zelle

9. Durchführung der Schritte 6 bis 8 für alle anderen Elemente

ZWISCHENERGEBNIS: 1 Tabelle mit 20 Spalten (X-Koordinate; Y-Koordinate; Cr, $\mathrm{Cu}, \mathrm{Fe}, \mathrm{Ni}, \mathrm{Pb}, \mathrm{Ti}, \mathrm{V}$ und Zn-Schätzwerte und metallspezifische Indizes; Raumklasse; Erhebungsjahr) und 122.889 Zeilen

10. Bildung eines Mittelwertes über alle 8 metallspezifischen Akkumulationsindizes pro Zeile (= standortspezifische und Kampagnen übergreifende Akkumulationsindizes)

11. Bildung je eines Medians über

(a) alle standortspezifischen und Kampagnen übergreifenden Akkumulationsindizes aus Schritt 10

(b) die jeweils einer Raumklasse und einem Erhebungsjahr zuzuordnenden standortspezifischen und Kampagnen übergreifenden Akkumulationsindizes aus Schritt 10

12. Berechnung des Quotienten (b - a / a x 100) [\%] pro Raumklasse

ENDERGEBNIS: 1 Tabelle mit 21 Spalten (X-Koordinate; Y-Koordinate; Cr, Cu, Fe, Ni, Pb, Ti, V und ZnSchätzwerte und metallspezifische Indizes; Raumklasse; Akkumulationsquotient; Erhebungsjahr) und 122.889 Zeilen

13. Import der Tabelle ins GIS und kartographische Darstellung des Quotienten aus Schritt 12

Kasten 2: Naturräumliche Differenzierung des Kampagnen integrierenden Multi-Metall-Akkumulationsindexes Natural order classification applied in the survey on integrative multi-metal accumulation indices Différenciation spatiale naturelle de l'index intégrateur des campagnes d'accumulation multimétallique

differenziert abzubilden und zu interpretieren. Mit Hilfe von GIS-Techniken werden weiterhin Hot Spots der Metallakkumulation lokalisiert.

Weiterführende Arbeiten sollten sich vor allem auf die Optimierung des Moos-Monitoring-Messnetzes 2005 konzentrieren. Die vorgestellten Hot Spots sind ein erster Ansatzpunkt, das Moos-MonitoringMessnetz gezielt zu verdichten. Unter Verwendung weiterer multivariater Analysemethoden sollte die in Kapitel 2.2 vorgestellte landschaftsökologische Raumgliederung dazu verwendet werden, die räumliche Verteilung der Moos-Monitoring-Standorte zu optimieren. Im Sinne der häufigkeitsstatistischen Landschaftsrepräsentanz nach ScHRöDER et al. (2001) sollten die Monitoring-Standorte flächenproportional auf die Ökoregionen verteilt werden. Mit Hilfe nachbarschaftsanalytischer Verfahren kann weiterhin eine quantitative Beschreibung der räumlichen Umgebungsstruktur von Rasterzellen in Bezug auf Merkmale wie Landnutzung und naturräumliche Ausstattung (raumstrukturelle Landschaftsrepräsentanz; SCHRÖDER et al. 2001) vorgenommen werden. Unter Berücksichtigung beider Repräsentanzkriterien kann das Moos-Monitoring-Messnetz gezielt ergänzt bzw. ausgedünnt werden. 


\section{Literatur}

BAILEY, R.G. (1996): Ecosystem geography. - New York, Berlin, Heidelberg: Springer-Verlag.

Bohn, U., Neuhäusl, R., Gollub, G., Hettwer, C., Neuhäuslová, Z., Schlüter, H. \& H. Weber (2003): Karte der natürlichen Vegetation Europas. - MünsterHiltrup: Landwirtschaftlicher Verlag.

Breiman, L., Friedman, J., Olshen, R. \& C. Stone (1984): Classification and regression trees (CART). Pacific Grove: Wadsworth.

Buse, A., Norris, D., Harmens, H., Büker, P., AshenDEN, P. \& G. Mills (2003): Heavy Metals in European Mosses. 2000/2001 Survey. - UNECE ICP Vegetation, Bangor, UK.

FräNZLE, O. (1998): Grundlagen und Entwicklung der Ökosystemforschung. - In: Fränzle, O., Müller, F. \& W. SChröDER (Hrsg.): Handbuch der Umweltwissenschaften. Grundlagen und Anwendungen der Ökosystemforschung. - Landsberg am Lech: ecomed: 1-24.

FränZle, O., Straskraba, M. \& S.E. Jørgensen (1995): Ecology and ecotoxicology. - = Ullmann's Encyclopedia of Industrial Chemistry Vol. B7, Weinheim: 19-154. Hennings, V. (2000): Methodendokumentation Bodenkunde. Auswertungsmethoden zur Beurteilung der Empfindlichkeit und Belastbarkeit von Böden. - = Geologisches Jahrbuch, Sonderhefte, Reihe G, Heft SG 1, Hannover.

Herpin, U., Lieth, H. \& B. Markert (1995): Monitoring der Schwermetallbelastung in der Bundesrepublik Deutschland mit Hilfe von Moosanalysen. - = UBA-Texte 31/95, Berlin.

Hornsmann, I. (2003): Eine ökologische Raumgliederung für Schleswig-Holstein mit dem Klassifikationsverfahren Cart. - Unveröffentlichte Diplomarbeit, Institut für Umweltwissenschaften, Hochschule Vechta.

KLIJN, F. (1997): A hierarchical approach to ecosystems and its implications for ecological land classifications with examples of ecoregions, ecodistricts and ecoseries of the Netherlands. - Wageningen: Ponsen \& Looijen.

Marks, R., Müller, M.J., Leser, H. \& H.J. KlinK (Hrsg.) (1989): Anleitung zur Bewertung des Leistungsvermögens des Landschaftshaushalts. $-=$ Forschungen zur Deutschen Landeskunde 229, Trier.

Meynen, E., Schmithüsen, J., Gellert, J., Neef, E., Müllerminy, H. \& J.H. Schultze $(1953,1962)$ : Handbuch der naturräumlichen Gliederung Deutschlands. - 2 Bde., Bad Godesberg: Bundesanstalt für Landeskunde und Raumforschung.

MucHA, H.J. (1994): Clusteranalyse, automatische Klassifikation. - In: SCHRöder, W., VetTER, L. \& O. FräNZLE (Hrsg.): Neuere statistische Verfahren und Modellbildung in der Geoökologie. - Braunschweig, Wiesbaden: Vieweg: 109-127.

MüLLER, P. (1981): Arealsysteme und Biogeographie. Stuttgart: Verlag Eugen Ulmer.
NRIAGU, J.O. \& J.M. PACYNA (1988): Quantitative assessment of worldwide contamination of air, water and soils by trace metals. - In: Nature 333: 134-139.

OhNSORGE, F.K. \& M. Wilhelm (1991): Zinc. - In: Merian, E. (ed.): Metals and their compounds in the environment. Occurence, analysis and biological relevance. - Weinheim, New York: VCH: 1309-1342.

OlEA, R.A. (1999): Geostatistics for Engineers and Earth Scientists. - Boston, Dordrecht, London: Kluwer Academic Publishers.

PeICHL, L. (2001): Umweltindikatoren für Immissionswirkungen. - In: Umweltwissenschaften und Schadstoff-Forschung. Zeitschrift für Umweltchemie und Ökotoxikologie 13, 3: 130-138.

PESCH, R. (2003): Geostatistische und multivariat-statistische Analyse der Ergebnisse des Moos-Monitorings 1990, 1995 und 2000 zur Ableitung von Indikatoren für atmosphärische Metalleinträge in Deutschland. Unveröffentlichte Dissertation, Institut für Umweltwissenschaften, Hochschule Vechta.

RENNERS, M. (1991): Geoökologische Raumgliederung der Bundesrepublik Deutschland. - = Forschungen zur deutschen Landeskunde 235, Trier.

SchmidT, G. (2002): Eine multivariat-statistische Raumgliederung für Deutschland. - Unveröffentlichte Dissertation, Institut für Umweltwissenschaften, Hochschule Vechta.

SCHRÖDER, W. (1994): Regionalisierung in den Geowissenschaften. - In: SCHRÖdER, W., VetTER, L. \& O. FRÄNZLE (Hrsg.): Neuere statistische Verfahren und Modellbildung in der Geoökologie. - Braunschweig, Wiesbaden: Vieweg: 17-30.

Schröder, W., Schmidt, G., Pesch, R., Matejka, H. \& T. ECKSTEIN (2001): Konkretisierung des Umweltbeobachtungsprogrammes im Rahmen eines Stufenkonzeptes der Umweltbeobachtung des Bundes und der Länder. Teilvorhaben 3. - = Abschlussbericht FuEVorhaben 29982 212/02, Berlin: Umweltbundesamt.

Schröder, W., Anhelm, P., Bau, H., Matter, Y., Mitze, R., Mohr, K., Peichl, L., Peiter, A., Peronne, T., Pesch, R., Roostai, H., Roostai, Z., Schmidt, G. \& U. Siewers (2002): Untersuchungen von Schadstoffeinträgen anhand von Bioindikatoren. Aus- und Bewertung der Ergebnisse aus dem Moos-Monitoring 1990/91,1995/96 und 2000/01. - = Abschlussbericht, 4 Bde., FuE-Vorhaben 20064 218, Berlin: Umweltbundesamt.

Schulz, J. (2000): Handbuch der Ökozonen. - Stuttgart: Verlag Eugen Ulmer.

SiEWERS, U. \& U. HeRPIN (1998): Schwermetalleinträge in Deutschland. Moos-Monitoring 1995. - = Geologisches Jahrbuch, Sonderhefte, Heft SD 2, Stuttgart: Gebrüder Borntraeger Verlagsbuchhandlung.

Siewers, U., Herpin, U. \& S. Strassburger (2000): Schwermetalleinträge in Deutschland. Moos-Monitoring 1995. Teil 2. - = Geologisches Jahrbuch, Sonderhefte, Heft SD 3, Stuttgart: Gebrüder Borntraeger Verlagsbuchhandlung. 
Steinhardt, U. \& M. VolK (Hrsg.) (1999): Regionalisierung in der Landschaftsökologie. - Stuttgart, Leipzig: Teubner.

Stevens, S.S. (1946): On the Theory of Measurement. - In: Science 103: 677-680.

UNECE (United Nations Economic Commission for Europe, Convention on Long-range Transboundary Air Pollution) (2001): Monitoring of Atmospheric Heavy Metal Deposition in Europe Using Bryophytes. Experimental Protocol 2000/2001 Survey. - Bangor, UK.

Weustermann, P. (2003): Eignung und Verfügbarkeit von Daten für die ökosystemare Umweltbeobachtung in Schleswig-Holstein am Beispiel der potenziellen Grundwassergefährdung durch Schwermetalle. - Unveröffentlichte Diplomarbeit, Institut für Umweltwissenschaften, Hochschule Vechta.

White, I.D., MotTershead, D.N. \& S.J. Harrison (1984): Environmental Systems. - London: George Allen \& Unwin.

\section{Zusammenfassung: Geographische Umweltmessnetz- analyse und -planung}

Der Artikel behandelt Verfahren für die weitgehende Zusammenfassung von Umweltdaten sowie die Kartierung der von ihnen abgebildeten räumlichen und zeitlichen Differenzierungen. Als Beispiel dienen Daten über die 1990, 1995 und 2000 gemessene Akkumulation von $\mathrm{As}, \mathrm{Cd}, \mathrm{Cr}, \mathrm{Cu}, \mathrm{Fe}, \mathrm{Hg}, \mathrm{Ni}, \mathrm{Pb}, \mathrm{Sb}, \mathrm{Ti}, \mathrm{V}, \mathrm{Zn}$ in Moosen an bis zu 1028 Standorten in Deutschland. Die Fülle der Messdaten wird stufenweise komprimiert: Die stoffliche Differenzierung wird durch einen nominal und einen ordinal skalierten Index reduziert. Die beiden Indizes integrieren die aus den Messdaten aller Metalle berechneten Flächeninformationen. Die räumlichen Trends werden auf die Klassen einer landschaftsökologischen Raumgliederung reduziert. Die Lokalisierung von Hot Spots der Metallakkumulation bietet Ansatzpunkte für eine Optimierung des Moos-Monitoring-Messnetzes 2005.

\section{Abstract: Geographical environmental monitoring net analyses and planning}

The UNECE Metals in Mosses survey provides data on the accumulation of metals in naturally growing mosses throughout Europe. Based on research in Germany in close to 1028 sites, this article concentrates on the further development and application of data analysis methodology necessary for a comprehensive interpretation of spatial and temporal trends related to metal accumulation in mosses. The sampling of mosses and their chemical analysis with relation to $\mathrm{Cr}, \mathrm{Cu}, \mathrm{Fe}$,
$\mathrm{Ni}, \mathrm{Pb}, \mathrm{Ti}, \mathrm{V}$ and $\mathrm{Zn}$ content in 1990,1995 and 2000 are summarised briefly. Due to the distinct autocorrelation structures detected in variogram analyses, spatial areas were defined according to Kriging. The cluster analyses of z-transformed estimation data resulted in a nominal multi-element index that indicates regional metal accumulation types over time. Percentile statistics were applied to calculate an ordinal scaled multielement accumulation index, allowing for the definition of multivariate statistically defined ecoregions spatially differentiated over time. Hot spots of metal accumulation could also be defined with the help of GIS techniques. These techniques could contribute towards optimising the monitoring net in the future.

\section{Résumé: Analyse et planification géographiques du réseau de mesure environnemental}

Le présent article traite des processus consacrés à l'établissement d'une vaste synthèse de données environnementales ainsi qu'à la cartographie des différenciations spatiales et temporelles qui en résultent. Sont présentées comme exemples les données collectées en 1990, 1995 et 2000, relatives au mesures d'accumulation d'As, de $\mathrm{Cd}, \mathrm{Cr}, \mathrm{Cu}, \mathrm{Fe}, \mathrm{Hg}, \mathrm{Ni}, \mathrm{Pb}, \mathrm{Sb}, \mathrm{Ti}, \mathrm{V}, \mathrm{Zn}$, dans les mousses de 1028 endroits adéquats en Allemagne. Le nombre imposant de données de mesures est comprimé progressivement: la différenciation des éléments est réduite par un indice d'échelle nominal et un indice d'échelle ordinal. Les deux indices intègrent les informations sur les surfaces calculées à partir des données de mesures relatives à l'ensemble des métaux. Les tendances spatiales sont réduites aux classes correspondant à une structure spatiale d'écologie paysagère. La localisation de «hot spots» de l'accumulation métallique offre des points d'appui à une optimisation du réseau de mesure du «monitoring» de mousse.

Dr. Roland Pesch, Prof. Dr. Winfried Schröder, Institut für Umweltwissenschaften, Abt. Landschaftsökologie, Hochschule Vechta, Oldenburger Strasse 97, D-49377 Vechta, Deutschland.

e-mail:

rpesch@iuw.uni-vechta.de

wschroeder@iuw.uni-vechta.de

\section{Manuskripteingang/received/manuscrit entré le} 10.3.2004

Annahme zum Druck/accepted for publication/accepté pour l'impression: 20.6.2005 